\title{
Il friulano: storia e usi letterari
}

\author{
Stefano Magni
}

\begin{abstract}
L'articolo parla della formazione del friulano ed esamina i suoi usi linguistici e letterari. Per comprendere il processo di caratterizzazione linguistica si è partiti da una puntualizzazione storico-geografica, completata da un'analisi storico-linguisitca. Si è accennato ai problemi dell'unità interna del friulano, dell'appartenenza al gruppo «retoromanzo», del rapporto con le lingue limitrofe. Per concludere si è presentato il panorama letterario friulano, il suo rapporto con la cultura locale e all'opposto il tentativo di emancipazione artistica.
\end{abstract}

Parole chiave: Storia del Friuli, identità del friulano, usi letterari del friulano.

\begin{abstract}
The article refers to the formation of Friulano and examines its linguistic and literary use. In order to understand the process of linguistic characterisation, the essay starts with an historico-geographical description, completed with an historico-linguistic analysis. Mention is made of the problems of the internal unity of Friulano, of its pertaining to the «retoromanzo" group, of its relationship with limitroph languages. In conclusion, the article sets out the literary panorama for Friulano, its relationship with the local culture and, in contrast to this, the attempts at artistic emancipation.
\end{abstract}

Key words: The history of Friuli, the identity of Friulano, the literary.

Fontana di aga dal me paìs. A no è aga pì fres-cia che tal me paìs. Fontana di rustic amòur. 
Xavier Lamuela nel suo Català, occità, friülà: llengües subordinades i planificaciò lingüistica, si esprime sul friulano in questi termini:

El ciutadà català de cultura mitjana ha sentit a parlar del reto-romànic com d'una de les llengües romàniques i sol tenir una vaga idea que és parlat a Suïssa i que allà és objecte d'una consideració especial: ve't aquí tot. Aquí no és el lloc d'entrar en la qüestió [...] de la unitat del reto-romànic. Ens bastarà saber que [...] el friülà és, amb gran diferència, la unitat major pel que fa a la base demogràfica —més de mig milió de parlants $[\ldots]^{1}$

Partendo da questa citazione posso provare a chiarire l'identità geografica e storica del Friuli, la sua complessità linguistica e la relazione che intrattiene con il retoromanzo, il latino, l'italiano e le altre lingue geograficamene limitrofe, presentandone la diffusione e gli usi artistici, soprattutto recenti.

Il Friuli ${ }^{2}$ occupa l'estremo Nord Est dell'Italia, confinando a Nord con l'Austria, a Est con la Slovenia, a Ovest con il Veneto e lambendo a Sud il mare Adriatico. Al centro la regione è attraversata dal fiume Tagliamento che crea la divisione interna "di qua dall'acqua e di là dall'acqua» che si manifesta anche in due grandi varietà linguistiche.

Il primo insediamento stabile in Friuli si può attestare con una certa sicurezza tra il v e il III secolo a.C., in seguito all'immigrazione della tribù celtica dei Carnunti, proveniente dalla Francia, che occupò la regione tra il Livenza e il Carso, dando il nome alla Carnia, alla Carinzia (in Austria), alla Carniola (in Slovenia). In precedenza è attestato il passaggio dei Veneti, anche se poco si sa del loro stanziamento.

La comparsa della latinità in Friuli risale al 181 a.C., con la fondazione della colonia di Aquileia. La posizione avanzata della colonia ne indicò subito la funzione di baluardo e questa sarà per lungo tempo la sua destinazione. I ricchi coloni romani occupavano una vasta fascia di terreno nella pianura più prossima al mare, coabitando con la massa più numerosa dei celto-carni e mettendo le basi della cultura romano-celtica che darà l'avvio all'identità della regione. Importante da questo punto di vista la persistenza in tutta la zona della lingua celtica anche in epoca romana.

Con il diffondersi della cristianità, Aquileia diventò sede vescovile e l'importanza del centro religioso marcherà la storia futura della regione, facendo gravitare intorno a sé per secoli la vita culturale, politica e amministrativa. Contemporaneamente assunse importanza il Forum Julii, così chiamato in onore di Giulio Cesare, località che cambierà il suo nome in Cividale e da cui deriverà il nome Friuli. La sorte di Aquileia e della regione intera decadde con

1. Xavier Lamuela, Català, occità, friülà: llengües subordinades i planificaciò lingüistica, Barcelona: Quaderns Crema, 1987, p. 175.

2. Come principali riferimenti bibliografici per una storia del Friuli si veda: Giuseppe FraNCescato, Fulvio Salimbeni, Storia, lingua e società in Friuli, Udine, 1976; Gian Carlo Menis, Storia del Friuli, Udine, 1969; Pio Paschini, Storia del Friuli, Udine, 1936; Pier Silverio LEICHT, Breve storia del Friuli, Udine, 1923. 
quella dell'Impero Romano; dopo il 476 d.C. si successero le invasioni barbariche che trovarono facile accesso alla penisola passando attraverso le dolci colline che marcano a Est il confine con la Slovenia. Solo nel 568 d.C. i Longobardi, di stirpe germanica, entrarono non con soli fini di razzia, ma per realizzare un solido insediamento che si integrerà con le realtà preesistenti.

Dopo i Longobardi fu Carlo Magno nel 776 a prendere possesso dei territori in nome dei Franchi fino a quando una serie di invasioni ungare nella prima metà del 900 mise in ginocchio la regione. Il lungo periodo di instabilità fu concluso con l'inizio della dominazione germanica della famiglia di Sassonia. Di fronte a questi cambiamenti di potere, l'unica realtà che mantenne una certa consistenza fu il patriarcato di Aquileia che apparì anche agli stessi imperatori sassoni come il riferimento politico più sicuro della zona. Grazie a ciò, da questo momento il Friuli visse la sua massima esperienza autonomistica e, isolato dal resto dell'Italia, potè conservare per secoli la sua lingua originale, ponendo le basi della sua identità.

Nel 1420 Venezia ebbe la meglio sulle truppe imperiali e pose termine all'esperienza del patriarcato, occupando la quasi interezza della regione, ma lasciando comunque anch'essa una certa indipendenza ai «buoni e fedeli friulani».

Con l'arrivo di Venezia, a livello sociologico e linguistico, è importante segnalare la venetizzazione dei centri più importanti che passarono dalla parlata friulana ad una veneta, retaggio che è stato tramandato fino ad oggi.

Venezia, però, non riuscì ad annettere Trieste e Gorizia che restarono in area germanica e che ritorneranno italiane solo alla fine della Prima Guerra Mondiale. Comunque l'unità linguistica fu mantenuta anche al di là del confine. Gorizia è linguisticamente parte dell'area friulana, Trieste lo fu fino all'inizio del XIX secolo.

Nei secoli XVI-XVIII la cultura friulana orbitava intorno ai due grossi centri: da un lato l'area germanica e dall'altro quella veneta, nettamente fratturate, fino a quando, nel 1797, furono le armate di Bonaparte ad annettere la regione, per farne subito terra di scambio, ovvero rendendola lo stesso anno all'Austria in cambio della Lombardia e di parte dei Paesi Bassi con quel trattato di Campoformio che indignò molti nazionalisti italiani tra i quali Foscolo. Fino al 1813 il Friuli fu palleggiato tra le due potenze e solo con la Restaurazione fu annesso all'Austria per restarvi fino al 1866 quando, attraverso i moti del Risorgimento che portarono alla formazione dell'Italia contemporanea, tornò ad essere italiano, con l'eccezione, come detto, di Gorizia e Trieste.

In epoca contemporanea la regione ebbe a soffrire il peso maggiore del primo conflitto mondiale che si combattè lungo i suoi confini orientali e visse tragicamente la disfatta di Caporetto dell'ottobre '17 con un anno di nuova sudditanza austriaca. Pesante fu la sorte anche durante la Seconda Guerra Mondiale, soprattutto dopo l'8 settembre '43, con l'annessione diretta al Terzo Reich come provincia adriatica (Adriatisches Künstenland) e con l'ultima «invasione», quella dei Cosacchi, alleati di Hitler, cui fu promessa la regione (Kosakenland) in cambio del controllo della particolarmente vigorosa lotta partigiana. 
Nel dopoguerra la posizione di confine con il comunismo (Jugoslavia) fece del Friuli un luogo massicciamente militarizzato. Nel pordenonese è tuttora stanziata la base americana più grande d'Europa e nella stessa zona, a testimoniare lo sfruttamento del territorio e delle risorse di una regione di tradizione povera, si consumò nel 1963 la catastrofe della diga del Vajont. Nello stesso anno venne però accordato lo statuto di Regione Autonoma e il capoluogo fu stabilito a Trieste. L'ultimo episodio saliente fu, nel 1976, il devastante terremoto che rase al suolo interi villaggi segnando una frattura con la vecchia cultura e minacciandone la sopravvivenza stessa. ${ }^{3}$

Queste determinazioni storiche ci servono per identificare il popolo friulano, determinarne la sua individualità in rapporto all'Italia. Il sentimento della Patria friulana, che risiede nella lunga esperienza del patriarcato di Aquileia e nell'autoctona mescolanza di cultura celtica, latina, longobarda, dà come immagine del popolo friulano un risultato piuttosto eclettico che pare emergere chiaramente anche a livello linguistico. Già dal '500, infatti, si parla di «sermo varius», di una parlata che «risuona [...] quasi di tutte le lingue straniere» (Marcantonio Sabellico, De Vetustate Aquileiae) e di una lingua in cui «hanno corso le parlate di quasi tutto l'universo, il che non è toccato a nessun'altra lingua» (Antonio Franceschinis, De Carnica Regione illustratio). Il giudizio è quantomeno sorprendente: il friulano è visto come prototipo ed emblema di mescolanza, come l'apice raggiunto dalle possibilità combinatorie della linguistica.

Un giudizio particolarmante indovinato mi pare quello di Paul Fabre (Le patrimoine de l'église romaine dans les Alpes Cottiennes, XIX sec.) «Il Friuli è una regione a sé, distinta dalle altre regioni nominate in precedenza perché non parla la lingua latina, né la slava, né la tedesca, ma possiede un suo idioma peculiare. Partecipa tuttavia più della lingua latina che di qualunque altra lingua vicina». ${ }^{4}$

Come primo elemento deduciamo una unicità del Friuli rispetto alle regioni confinanti. In secondo luogo i termini dell'identificazione sono linguistici. La parlata locale è diversa da tutte quelle confinanti, pur essendo sicuramente neolatina piuttosto che germanica o slava. ${ }^{5}$

3. Questa tendenza, probabilmente insita nel corso storico stesso, è stata violentemente accelerata dal cataclisma. In quel momento finisce in Friuli la millenaria civiltà contadina che per molti secoli aveva caratterizzato la cultura della popolazione e che, rispetto ad altre regioni italiane, soprattutto del Nord Italia, si era conservata più a lungo. Questo elemento ci sarà utile per comprendere la produzione artistica e letteraria attuale della regione, che ha giocato continuamente su un confronto reiterato e a volte patetico con la tradizione.

4. Per un riassunto delle differenti posizioni si veda: Rienzo Pellegrini, Tra lingua e letteratura: per una storia degli usi scritti del friulano, Udine: Casamassima, 1987, p. 24-27 e p. 111-112.

5. I confini dettagliati del friulano sono delineati nell'imponente Atlante Storico-Linguistico-Etnografico-Friulano (ASLEF) che in modo sistematico analizza parlate e toponimi della regione. I confini generali dati per delimitare l'area linguistica sono: il fiume Livenza ad Occidente, ad Oriente "è ben noto che il friulano o una sua varietà si estendeva sino a Trieste ed a Muggia». L'ASLEF suddivide inoltre questo territorio in quattro grandi aree, corrispondenti alle varietà: 1) centrale, 2) orientale, 3) occidentale e 4) carnica; ma «[...] è divisione 
All'interno della regione, comunque, l'espressione grafico-linguistica presenta una situazione molto variegata. Le ragioni dello smembramento sono molte: di ordine storico, geografico e sociologico. L'influenza del veneto, per esempio, agisce bilateralmente. Da un lato c'è una sua forte influenza sui dialetti della campagna, maggiore quanto più ci si avvicina al confine occidentale. Da un altro lato Venezia irruppe linguisticamente nei centri principali della regione creando quella frattura città-campagna cui ho già accennato e su cui si sono espressi molti friulanisti.

G.B. Pellegrini ha avuto modo di notare come «nei secoli XI-XIV [...] Il veneto diviene via via il linguaggio quotidiano delle classi borghesi, dei nobili e delle famiglie più abbienti e cittadine [...] s'insinua nei centri cittadini e si affianca al friulano per eliminare totalmente quest' ultimo $[\ldots]{ }^{6}{ }^{6}$

I caratteri delineati sono evidenti: il veneto è assunto come prima lingua da borghesi e nobili e nel contesto cittadino (ma Udine aderì a questa convenzione solo nel XX secolo); il friulano è ridotto a idioma familiare, limitato ad un ambito ristretto geograficamente (la regione) e socialmente (famiglia, popolo e campagna). Questo fenomeno crea a tutt'oggi nella regione delle aree venetofone e non-friulanofone. Una dinamica simile avviene lungo il confine orientale, infatti, come ricorda G.B. Pellegrini, « $\mathrm{E}$ ben noto che la regione di Trieste con Muggia (e forse [...] ivi compresa Capodistria), rappresentava ancora alla metà del secolo passato una propaggine dell'area linguistica friulana in terra giuliana in via di totale assorbimento ad opera del dialetto veneto». ${ }^{7}$

Il glottologo bellunese identifica un idioma antico, il tergestino che possiamo equiparare al friulano, rimpiazzato da uno di tipo veneto, il triestino.

Il vorticoso aumento della popolazione a Trieste e l'estendersi dell'abitato, specie dopo la proclamazione della cittadina a porto franco, l'affluenza dei forestieri da ogni paese d'Italia e d'Europa, il formarsi di una coscienza nazionale molto sentita, rese assolutamente inefficiente ed insufficiente l'uso del vecchio dialetto tergestino che venne via via ripudiato e ritenuto di rango inferiore soprattutto nelle classi dei borghesi e dei numerosi commercianti, e, in un

essenziale quella che riconosce due varietà fondamentali [...] e ciò̀: 1) il friulano aquileiese [...] sulla sinistra del Tagliamento e il friulano concordiese sulla destra [...]». Pellegrini parla di «varianti più importanti». All'interno di queste si può ipotizzare una complessa mappa piuttosto maculata di varianti inferiori la cui forza centrifuga non distrugge però la realtà della stessa matrice celto-romanica e l'unità complessiva rispetto alle zone limitrofe (Austria, Slovenia, Italia); tanto che Pellegrini accetta una "unità linguistica friulana" che ricopre grosso modo l'attuale Regione Friuli-Venezia Giulia». Cfr. Giovanni Battista PeLLEGRINI, "ASLEF, ragioni dell'opera e programma», in Introduzione all'atlante storico-linguistico-etnografico-friulano (ASLEF), Udine: Istituto di glottologia dell'Università di Padova/ Istituto di Filologia Romanza della Facoltà di Lingue e Letterature Straniere di Trieste con sede a Udine, 1972, p. 13-15.

6. Giovanni Battista PELLEGRINI, «ASLEF, ragioni dell'opera e programma», op. cit., p. 19-20. Si veda inoltre Giuseppe FranCESCATO, «Il dialetto veneto di Udine. Schizzo storico-fonetico", in Atti dell'Accademia di Udine, serie 6, vol. 13, 1954-1957.

7. Giovanni Battista PeLLeGRINI, «Tra friulano e veneto a Trieste», in Saggi sul ladino dolomitico e sul friulano, Bari: Adriatica editrice, 1972, p. 406. 
secondo tempo, anche tra il popolo e tra le poche famiglie nobili, in questo caso singolarmente attaccate alla tradizione avita. ${ }^{8}$

Il friulano, dunque, in primo luogo è inefficiente in una comunità espansa, poiché incomprensibile dai forestieri; dall'altro è sentito come lingua povera, evidentemente legata al semplice mondo contadino, rifiutata per questo dalla nuova borghesia commerciale.

Anche storicamente sul friulano pesa il giudizio di lingua popolare, spesso descritta come poco piacevole all'udito. Questa etichetta compare già dal primo riconoscimento dell'individualità del friulano. Nel De vulgari eloquentia, Dante usa termini piuttosto critici per descriverlo: «Post hos aquilegienses et Ystrianos cribremus, qui Ces fas-tu ? crudeliter accentuando eructuant. Cumque hiis montanina omnes et rusticanas loquelas eicimus [...]». ${ }^{10}$

In quel «crudeliter» si racchiude molta della considerazione che uno straniero doveva avere della parlata friulana. ${ }^{11}$ Nonostante i giudizi di gusto, comunque, c'è il riconoscimento di un idioma indipendente dal latino, dal veneto e dal toscano e poi dall'italiano, come anche dalle lingue d'oltre confine. Per il cittadino friulano questa individualità è un fatto culturalmente importante, come ricorda G.B. Pellegrini: "[...] è sempre di attualità, specie per i friulani, non certo per gli scienziati [...] un giudizio a proposito della loro parlata, se cioè essa debba essere considerata una «lingua» o un "dialetto»». ${ }^{12}$ Il tono dello studioso è piuttosto polemico: l'appartenenza del friulano al sistema delle lingue piuttosto che a quello dei dialetti è questione che interessa i friulani, non gli scienziati. Forse perché, come ha sottolineao Tito Maniacco, poter parlare di lingua può voler dire riconoscersi in una patria, altra rispetto all'Italia. E il cit-

8. Ibid., p. 410.

9. Il Pellegrini osserva anche come la formazione del triestino abbia dovuto procedere per ipercaratterizzazione nel rifiuto, ovvero formandosi per opposto all'idioma precedente. Lo studioso nota infatti come nel triestino manchi l'inversione soggetto verbo nelle domande, tratto comune al friulano come a tutti i dialetti veneti.

10. Dante Alighieri, De vulgari eloquentia, I, XI, introduzione, traduzione e note di Vittorio Coletti, Milano: Garzanti, 1995 [1991], p. 30-31. Trad.: Quindi setacciamo Aquileiesi e Istriani che Ces fas-tu? eruttano con pronuncia crudissima e con loro sbattiamo via tutte le parlate montanare e campagnole $[\ldots]$

11. Anche altri pareri, tra i quali quelli del Sabellico e del Capretto segnalavano nel friulano delle sonorità spiacevoli, dure e poco musicali.

12. Giovanni Battista PellegrinI, "ASLEF, ragioni dell'opera e programma", op. cit., p. 16. Anche alcuni scrittori «locali» hanno espresso i loro pareri, tra essi Pasolini e Sgorlon. Pasolini ha detto del friulano ancora negli anni '40: "Non un dialetto italiano, ma neanche una lingua; è lì, il nostro friulano, a mezza strada, e forse spera ancora che qualcuno dei suoi figli lo innalzi e gli dia quella dignità che gli spetta» in Pier Paolo PASOLINI, «Dialetto, lingua, stile», in L'Academiuta friulana e le sue riviste, a cura di Nico NALDINI, Vicenza: Neri Pozza, 1994, p. 12. Più recentemente Sgorlon propende piuttosto per un declassamento del friulano: "Quando si scriveranno romanzi moderni in cui si parlerà di aerei, di calcolatori elettronici, ecc. allora sarò più disposto a credere che il friulano sia una lingua, ma credo che questo non accadrà mai. Perciò il friulano è un linguaggio dialettale " (Liana NISSIM, Sgorlon teste insolente: materiali per un'ermeneutica e una dichiarazione poetica di Carlo Sgorlon, Udine: I quaderni del Gamajun, 1985, p. 141-142). 
tadino friulano vive in questa situazione ambivalente, per cui storicamente e linguisticamente sente un'indipendenza, ma sempre sotto l'egida di un potere più forte, emanato spesso dalla penisola italiana.

Il fatto stesso di rivendicare un'autonomia dipende forse dal fatto che essa è costantemente messa in dubbio da molteplici fattori: il sempre minor numero di parlanti friulano, l'italianizzazione anche delle aree agresti, un'indipendenza politica limitata.

L'indipendenza linguistica del friulano fu studiata dal glottologo goriziano Graziadio Isaia Ascoli che, nel 1873, pubblicava i Saggi Ladini con cui individuava su base tipologica un complesso di parlate marginali neolatine caratterizzate da un insieme di tratti che conferivano loro una determinata specificità all'interno della Romània.

Allora il termine "ladino» ${ }^{13}$ veniva ufficialmente ad identificare una fascia (discontinua) di parlate alpine che da alcuni territori della Rezia, attraverso l'area dolomitica, raggiungeva il Friuli e l'Istria. ${ }^{14} \mathrm{Ma}$ il vero senso di questa classificazione, l'omogeneità dell'area, i rapporti tra le tre parlate sono stati spesso discussi e definiti in modi differenti. Le obiezioni all'unità sono sostanzialmente di natura storica, lessicale e a volte si rifanno alla evidente incomprensione tra i parlanti di queste aree. ${ }^{15}$

Il friulano, comunque, come ricordava X. Lamuela, è tra queste lingue l'idioma più parlato. Si aggiunga anche che è quello con la tradizione letteraria più antica.

13. Il termine «ladino», introdotto nel 1832 da Haller, è stato ripreso dall'Ascoli; la voce «retoromanzo» risale invece al Gartner.

14. Si veda la Relazione della commissione esaminatrice sulla Memoria di Giovanni Battista Pellegrini e Paola Barbierato dal titolo Comparazioni lessicali retoromanze: complemento ai saggi ladini di G.I. Ascoli in Giovanni Battista Pellegrins e Paola BARBIERATO, Comparazioni lessicali retoromanze: complemento ai saggi ladini di G.I. Ascoli, Venezia: Istituto Veneto di Scienze, Lettere ed Arti, 1999.

15. A questo punto potrebbe risultare interessante una scorsa rapida alle caratteristiche linguistiche del friulano, per cercare di identificarlo in rapporto alle altre lingue, presentando i rapporti di continuità con le lingue latine o con quelle celtiche.

In comune con le parlate latine presenta: una maggioranza di voci provenienti dal lessico latino medievale; una struttura morfologica analoga a quella latina, ma semplificata dalle flessioni; una sintassi legata a quella latina nella sua forma più popolare.

In comune con le lingue celto-latine, soprattutto oltremontane, presenta: un largo sistema di troncamenti desinenziali, con uscite consonantiche; la conservazione della «\$» finale nei plurali e nelle seconde persone dei verbi; la conservazione dei gruppi consonantici formati da una muta più la liquida «l» $(g l, b l, c l, p l, f l)$; un certo numero di voci derivanti dall'antico celtico.

$\mathrm{Ma}$, a differenza della maggior parte delle lingue celto-romane ignora i suoni vocalici intermedi fra «i» e «u» (ü) e fra «o» e "e» "œ»; conserva la «a» tonica dei verbi di prima coniugazione; accoglie numerose voci d'origine germanica e qualche centinaio di provenienza slava.

A livello consonantico, uno dei tratti più salienti, è la pronuncia prepalatale (o mediopalatale) di «c» e «g», che danno «ć» e «"g».

A livello vocalico terrei soprattutto conto della distinzione quantitativa delle vocali e della ricchezza di forme dittongate. 
La sede non mi permette di tracciare una storia della letteratura friulana, per cui vorrei concentrarmi su alcuni fenomeni della sua tradizione scritta per finalizzarli in un'ottica precisa. Mi pare punto interessante di discrimine, infatti, l'ambivalenza del friulano che da un lato vive di una realtà e di una tradizione fortemente agresti che ne interpretano il sapore più genuino della lingua, dall'altro aspira a un'elevazione universalmente poetica. Come ho detto, infatti, il friulano non ha avuto mai la dignità di linguaggio burocratico e politico, è stato emarginato dalle aree cittadine nobili e borghesi ed è restato marginalizzato nelle aree agricole. Lo scrittore che consapevolemente ne ha fatto uso, quindi, si è confrontato con una duplice dimensione, cercando da un lato di esprimere tutto quello che il friulano portava in sé in modo intrinseco, quindi una patina rurale e spontanea, dall'altro di farne strumento per ottenere i risultati artistici del modello toscano-italiano. Già la prima attestazione scritta del friulano, così antica da essere motivo di nobilitazione linguistica, esprime un certo sapore agreste. La ballata Piruç myo doç ${ }^{16}$ (1380) evoca allusioni villerecce, poiché di primo acchito al paragone floreale della «bella», tipico della lirica cortese, è sostituito il più carnoso riferimento al frutto del pero. La seconda ballata di cui si ha menzione Biello dumlo di valor (1416), appartiene al repertorio giullaresco. Il Soneto furlan (sec. XIV), pubblicato solo nel dopoguerra, a causa della sua ardimentosa metafora, è rusticamente licenzioso offrendo uno scoperto parallelismo tra i lavori della campagna e l'atto sessuale.

Con questa tradizione alle spalle, Pietro Capretto si trovò, nel 1484, a tradurre le Costituzioni della Patria del Friuli. È interessante notare come l'autore scelga per il volgarizamento non il friulano, ma il trevigiano, da intendere sicuramente in modo lato come veneto e in cui R. Pellegrini constata come «si innesti una buona dose di tratti linguistici toscani». ${ }^{17} \mathrm{E}$ ' significativo, comunque, che a questo periodo di sostanziale autonomia politica, nel momento di massimo splendore del patriarcato di Aquileia, risalgano i primi manoscritti in friulano.

16. Anche in questo caso bisognerebbe aprire una lunga parentesi per riassumere le varie ipotesi interpretative. Sinteticamente e in modo molto semplicistico, ignorando per esempio che piruç è maschile, e che al maschile accorda l'aggettivo, possiamo frettolosamente ipotizzare una traduzione del tipo «Peruccia mia dolce». Per un'esaustiva trattazione si veda Rienzo Pellegrini, Tra lingua e letteratura, op. cit., p. 56-62, in cui si ipotizzano le pertinenti alternative per cui piruç sarebbe meglio il frutto del biancospino e che allo stesso tempo la parola giocherebbe sul diminutivo del nome Pietruccia. Il dubbio relativo ad una certa identificazione piruç=pera sta nel linguaggio alto del componimento, anche se l'ambiguità potrebbe essere cosciente per l'autore.

17. Ibid., p. 99. In una epistola che funge da premessa alla traduzione, il Capretto spiega la sua rinuncia alla lingua toscana, perché oscura ai friulani, ma anche all'idioma friulano, perché non universale in tutto il Friuli e perché poco diffuso. Un buon compromesso gli pare, appunto, la lingua trevigiana. Il rifiuto del friulano pare tecnico, ovvero consiste nella mancanza di una tradizione scritta e che ove ce l'abbia risulta piuttosto popolare e a tratti giullarescoscabrosa, quindi inadatta all'alto scopo. Per ciò che concerne il tema dell'incomprensibilità del friulano all'interno degli stessi confini regionali, sono state avanzate ipotesi secondo cui questa lacuna non fosse geografica, ma socio-linguistica. Nel momento in cui il Capretto volgarizza le Costituzioni si può ipotizzare una forte componente di famiglie venetofone, sicuramente in corrispondenza dei borghi maggiori e delle classi più agiate. 


\section{In ogni caso il volgarizzamento del Capretto è una sorta di «atto manca-} to». La scelta, che abbiamo visto essere attentamente meditata, è pertanto simbolicamente un atto di rinuncia, un giudizio che colloca o forse costringe il friulano in ambito marginale e solo con un'ipotesi fantastica e assolutamente poco scientifica potremmo congetturare i possibili sviluppi della parlata davanti al gesto coraggioso di un traduttore che dal latino avesse intrapreso la soluzione più popolare.

Ma intanto il friulano, in letteratura, si forma poco a poco l'immagine di lingua dello scherzo spontaneo, del gioco paesano e semplice, attraverso un percorso che tocca i minori, ma che soprattutto coinvolge le due voci più rappresentative. Massimo esponente del '600 è Ermes di Colloredo, poeta in cui non è esclusiva, ma prevale la tematica rusticale ${ }^{18}$ alle volte traslata in una visione un po' edulcorata, in un mito bucolico della vita semplice e gradevole del contadino che può profittare dei piaceri della natura. I temi sono spesso satirici e fanno largo uso dell'obscenitas e la funzione spesso sociale del testo (creato sempre per un'occasione specifica, con un uditorio individuato) favoriscono il gioco, lo sberleffo, la satira.

La vena comico-satirica è raccolta dallo Zorutti (1792-1867) che è forse il più fulgido esempio di poesia friulana vista come gioco, lazzo, witz. Zorutti, che canonizza nell'uso letterario la variante del friulano centrale, diventa un paradigma linguistico. ${ }^{19} \mathrm{Ma}$ la sua grande fortuna a livello letterario è stata più volte messa in dubbio, proprio perché legata ad una facile vena ridente e ciarliera, interpretata con ottimo spirito dal personaggio-autore di cui si tramanda la battuta sagace e lo spirito acuto. Basti citare il severo giudizio di Domenico Del Bianco:

18. Rienzo Pellegrini, Tra lingua e letteratura, op. cit., p. 196.

19. Altro dibattito aperto e acceso è quello tra sostenitori e detrattori della «koinè» e non può essere sviluppato in questa sede con il necessario rispetto per tutte le opinioni; pare importante comunque ricordare che esso riguarda gli usi scritti, poiché «[...] il friulano scritto si è sviluppato generalmente sulla base delle varietà "centrali», facenti capo a Udine, centro politico, amministrativo e culturale della regione: e, come è ovvio, molti degli studi sul friulano si sono limitati a prendere in esame appunto la varietà rappresentata, in linea culturale di massima, dal friulano scritto (il quale aveva tra l'altro l'appoggio culturale e di prestigio della Società Filologica Friulana)» (Giuseppe FRANCESCATO, «La dittongazione friulana» [1959, p. 14-21], in Studi linguistici sul friulano, Firenze: Loescher, 1970, p. 15).

Francescato aggiunge poi che in ogni momento dello sviluppo della letteratura friulana gli pare di poter constatare un contrasto essenziale fra le tendenze unificatrici, variamente rappresentate, e la resistenza opposta dall'individualismo di vari scrittori che trovano la miglior espressione della loro vena poetica nella parlata nativa e particolare. Secondo Francescato nessuna autorità letteraria e linguistica nel corso dei secoli è riuscita mai a travolgere ogni resistenza locale e a imporre un modello di koinè adottato sull'intero territorio: «Possiamo tutt'al più parlare di una lingua letteraria che in certi periodi storici ha dominato più o meno il campo, mai interamente» (Giuseppe FRANCESCATO, "Per una storia del friulano letterario» [1959, p. 163-174], in Studi linguistici sul friulano, op. cit., p. 173). Il problema verte soprattutto sull'identificazione della koinè intorno agli scritti di Ermes di Colloredo e Pietro Zorutti che hanno raggiunto grande notorietà usando la stessa varietà linguistica.

$\mathrm{Al}$ contrario, a sostegno di una koinè friulana, si veda soprattutto Giuseppe MARCHETTI, «La koiné friulana attraverso i secoli», Ce Fastu, n. 26, 1950, p. 4-9 e "Per una koinè friulana», Sot la nape, n. 5, 2, 1955, p. 3-5. 
Zorutti $[\ldots]$ è più ammirato per le sue barzellette, per le sue più volgari corbellerie, che per le sue cose migliori. Non del tutto a torto è stato accusato di avere corrotto il buon gusto dei friulani. Essi sono da tanto tempo abituati a leggere nel loro dialetto poesiette facili, scorrevoli, frivole, satiriche ed umoristiche, che non domandano di meglio ai loro poeti. ${ }^{20}$

Zorutti riprende inoltre la stilizzazione arcadica che era stata anche del Colloredo, offrendoci quel modello di letteratura che comincia ad avere il sapore di friulano, sospeso tra leggerezza, satira e mito della semplicità contadina.

L'immagine della campagna come idillico quadretto lussureggiante perde consistenza con Caterina Percoto (1812-1887), ma il mondo rurale resta fulcro della pagina letteraria, mito di vita moralmente sana, anche se viene descritto con più corpo, con più realismo.

«Poeta contadino» è stato definito anche Celso Cescutti (1877-1966), ma anche in lui c'è un approfondimento di temi e mezzi espressivi che lo elevano verso l'assoluto. Fino ad arrivare alla reazione contro questa deleteria ${ }^{21}$ pratica poetica zoruttiana che venne promossa verso la fine del secolo da Pietro Bonini (1844-1905), il quale si sforzò di conferire al friulano un certo prestigio letterario, traducendo, tra l'altro, dalla Divina Commedia.

L'esperienza forse più importante del XX secolo è quella di Pasolini che mette il suo spirito creativo e innovatore al servizio della lingua "materna». ${ }^{22}$ Pasolini crea quella frattura culturale che getta le basi per una letteratura moderna in Friuli, battendosi contro la poetica della stucchevole tradizione, del focolare, della "polenta», contro «l'immoto mercato udinese»:

Non badare ai cruscaioli friulani sono ignoranti e faziosi, e vogliono ridurre lessico e sintassi friulani a documenti di un cretino, campanilistico moralismo. Vogliono che "polenta» rappresenti la morale della polenta, con questo bel sottinteso sociale: il friulano è contento di mangiar polenta, non chiede di meglio che mangiar polenta. ${ }^{23}$

La riscoperta vernacolare di Pasolini arriva alla morte del fascismo diventando gesto politico e sociale, contro le fissazioni accentratrici del regime che aveva ostacolato la pratica dei dialetti. Soprattutto, poi, l'uso del friulano come linguaggio letterario non nasce più dalla vena scherzosa, ma dalla sentita necessità di espressione e dall'orgogliosa affermazione della propria storicità linguistico-dialettale.

In seguito, in letteratura, la poesia friulana conta eccellenti firme, prestigiosi risultati artistici, anche se forse non ha mai raggiunto la sperimentazione che si è conosciuta negli anni '60-'70 in italiano o in altre lingue. Ma non si può

20. Domenico Del Bianco, Pagine Friulane, XVI, 1905-1906, p. 85.

21. Così Bonini stesso.

22. A proposito di Pasolini si parla di lingua materna in modo improprio, essendo la lingua della madre, appresa nei suoi soggiorni giovanili a Casarsa.

23. Pier Paolo PASOLINI, L'Academiuta friulana e le sue riviste, op. cit., p. 24-25. 
leggere la progressione del friulano come un'eterea ascesa verso l'Olimpo artistico. In realtà

I forti finanziamenti che arrivano dall'amministrazione regionale per la poesia, hanno creato una massa di letterati che si affermano solo nel contesto regionale senza riuscire e volere confrontarsi con le realtà nazionali e internazionali. Questi poeti scrivono in dialetto friulano (l'unica lingua a essere realmente finanziata), rimanendo ancorati a un mondo ormai scomparso, che tentano di ricreare attraverso affabulazioni idillico-nostalgiche oppure stilemi sotto-ermetici, in sostanza per mezzo di una vaghezza del sentire molto spesso stucchevole. $^{24}$

Se quindi a livello letterario il Friuli non è riuscito a liberarsi del tutto dalla "poetica della polenta» e anzi parte dell'espressione letteraria (e artistica in genere) ricade pesantemente in questo topos usatissimo e stantio, è pur vero che soprattutto negli ultimissimi anni lo standard di qualità della produzione artistica (e di rimando poetica) si è notevolmente emancipato dal giogo della tradizione contadina. A livello linguistico, poi,

[...] si è cercato nel Friuli un mezzo espressivo che non coincidesse con il friulano classico o «standard», ritenuto, magari a torto, troppo logoro a causa dei trascorsi letterari leggeri e fatui. Questa ricerca espressiva [...] ha generato nel Friuli una situazione linguistica assai complessa, con un friulano «lingua» contornato dai dialetti friulani, o almeno venato di dialettismi. Pertanto, nel Friuli oggi i dialetti sono in sottordine rispetto a un friulano scritto «sopradialettale» (arealmente centrale, si potrebbe dire), non facendo certo capo all'italiano né quelli, né questo; e possono quelli prestarsi, a loro volta, a prove letterarie. ${ }^{25}$

Ancora una volta la tradizione offre dall'artista un mezzo troppo compromesso con il fatuo sberleffo grassoccio e cerca una lingua paradossalmente artificiale per esprimere un sentimento poetico universale. Se in poesia, comunque, si sono avute molte esperienze interessanti, non altrettanto si può dire per la prosa, la cui produzione è significativamente assente, tanto è vero che se esistono antologie, raccolte e critiche della poesia friulana contemporanea di lingua ladina, nessun accenno si fa mai alla narrativa. ${ }^{26}$

I narratori friulani esistono, sono conosciuti ben al di là dei confini regionali, ma hanno scelto l'italiano per esprimersi. ${ }^{27}$ Anche parlando di argomenti locali questi autori hanno optato per la lingua nazionale. Sul tipo di scelta si

24. Alberto GarlinI, "Sulla poesia in Friuli», Perimmagine, anno XV, n. 1-2, inverno 20022003, p. 40-41.

25. Walter BELARDI e Giorgio FAGGIN, La poesia friulana del Novecento, Roma: Bonacci editore, 1987 , p. 13.

26. Spesso anche i concorsi letterari regionali prevedono una sezione per la poesia in friulano, ma al contrario un'altra sezione per la prosa in lingua italiana. È però di quest'anno la prima edizione del Premio Letterario Renato Appi, per la narrativa in friulano.

27. Tra i nomi più celebri ricordo Carlo Sgorlon, Elio Bartolini, Paolo Maurensig. 
esprime, con opinioni non sempre condividibili, Carlo Sgorlon che prima spiega come scrivere in friulano vorrebbe dire essere letto solo da poche centinaia di persone e poi aggiunge:

Ci sono scrittori che hanno questo destino, di essere letti soltanto dai friulani, ma i friulani conoscono già benissimo il Friuli, quindi gli scrittori in friulano sono superflui. Caso mai il mio compito è un altro, è quello di far conoscere il Friuli fuori dal Friuli, cioè agli italiani, e possibilmente anche all'estero. Il problema era questo: far conoscere il Friuli, la sua storia, i suoi costumi, i suoi miti, le sue leggende, la sua mentalità. ${ }^{28}$

Il risultato è che oggigiorno non si può parlare di un'esperienza narrativa friulana, se non a livelli veramente provinciali e amatoriali. D'altronde anche lo stesso Pasolini ci ha lasciato un testo teatrale in friulano (I Turcs tal Friûl), ma la stagione romanzesca lo vide sperimentare con la lingua delle borgate romane, abbandonando quella «materna».

In conclusione, il friulano è dunque ancora una lingua viva, con una produzione letteraria soddisfacente?

Sulla quarta di copertina di Contribution par une bibliografie (1945-1997), ${ }^{29}$ opera che raccoglie oltre mezzo secolo di pubblicazioni in lingua friulana, si legge con un certo orgoglio:

Oltre mezzo secolo di pubblicazioni, 1460 titoli, più di 400 scrittori: la lingua friulana offre pienamente la sua presenza di lingua di popolo, lingua di una identità che nel frattempo cerca nuove strade per mostrarsi.

Certo, la frase odora un po' di propaganda, tanto che all'interno i toni sono un po' più moderati e soprattutto nell'introduzione (jentrade) si sottolinea il parallelo tra richiesta e offerta libraria, per cui, senza un reale mercato, la produzione libraria non arriva alle soglie del costoso mondo editoriale ufficiale. ${ }^{30}$ $\mathrm{Ma}$, almeno da questo punto di vista, i finanziamenti per la salvaguardia delle lingue minoritarie dovrebbero incentivare la produzione. Si auspicano quindi autori di buona qualità che vogliano mettere il loro genio al servizio di una collettività linguistica fragilmente autonoma.

28. Liana Nissim, Sgorlon teste insolente, op. cit., p. 136-137.

29. Contribution par une bibliografie (1945-1997), Udine: Forum Editrice Universitaria Udinese, 1998.

30. Contribution par une bibliografie, op. cit., p. 9-10: "L'editorie e va in cubie cu la lenghe: se no si à un marcjât che al domande cun inxistence un prodot [...] lis publications a cirin une vie mancul ufficiâl di chê de cjase editore; [...]". 